\title{
Burned-Out Testicular Choriocarcinoma with Retroperitoneal and Liver Metastases
}

\author{
Woong Bin Kim', Sang Wook Lee', Ji Na Yun ${ }^{2}$ \\ Departments of ${ }^{1}$ Urology and ${ }^{2}$ Oncology-Hematology, Soonchunhyang University Bucheon Hospital, Soonchunhyang University College of Medicine, \\ Bucheon, Korea
}

\begin{abstract}
Spontaneous regression or the 'burned-out' phenomenon of testicular germ cell tumors is well-recognized but a rare clinical entity of which few reports exist. Tumors with the 'burned-out' phenomenon are found in metastatic sites, not in the testis. We report a 33-year-old patient who presented with liver choriocarcinoma and a retroperitoneal mass with the 'burned-out' phenomenon in the testis.
\end{abstract}

Keywords: Choriocarcinoma; Testicular neoplasms; Retroperitoneal mass

\section{INTRODUCTION}

The 'burned-out' phenomenon in germ cell tumors is defined as the presence of an extragonadal germ cell tumor with no evidence of a testicular neoplasm. This condition is different and less common than primary extragonadal germ cell tumors, which represent $2 \%-5 \%$ of adult germ cell malignancies. The origin of extragonadal germ cell tumors remains a matter of debate, and whether such tumors represent metastases of a primary testicular tumor or develop primarily at extragonadal sites is uncertain. Subsequently, Azzopardi et al. [1] firmly established evidence of spontaneous testicular germ cell tumor regression after examining autopsy material from patients who died of widely metastatic testicular germ cell tumors but lacked testicular masses.

We describe spontaneous regression of a primary testicular tumor with metastatic liver and retroperitoneal germ cell tumors, known as the 'burned-out' phenomenon.

\section{CASE REPORT}

A 33-year-old man presented with left upper quadrant pain and dyspepsia. The patient had a orchiopexy history due to bilateral cryptorchidism. An abdominal computed tomography (CT) scan showed a retroperitoneal mass of $10.7 \times 9.5 \times 16 \mathrm{~cm}$ abutting the aorta (Fig. 1). A second CT scan showed a rim-enhancing low-density lesion of the liver and dilatation of the left renal pelvocalyceal system due to the mass effect (Fig. 1). We then examined testicular tumor markers. The patient's serum $\beta$-human chorionic gonadotropin ( $\beta$-hCG) was beyond the normal range $(340.5 \mathrm{mIU} / \mathrm{mL})$, but his $\alpha$-fetoprotein $(\alpha-\mathrm{FP})$ level was normal. There was no abnormality in the physical examination of the scrotum. However, scrotal ultrasonography showed a heterogeneous echoic intratesticular mass in the left testis. We performed a radical left orchiectomy, and the histological examination showed a well-demarcated nodular scar with large aggregates of Leydig cells in a surrounding atrophied testis (Fig. 2). A sheet of large atypical cells with abundant eosinophilic cytoplasm and moderately pleomorphic nuclei was noted in the periphery of the nodular scar. A needle biopsy of the liver was conducted to exclude seminoma and other germ cell tumors. The histological analysis showed mononuclear and multinuclear tumor cells with abundant eosinophilic cytoplasm in a hemorrhagic and necrotic background (Fig. 3). The tumor cells were positive for pancytokeratin and hCG but negative for cytokeratin-19, vimentin, a-FP, and placental-like alkaline phosphatase. These findings were
(C) 2018 Soonchunhyang Medical Research Institute This is an Open Access article distributed under the terms of the Creative Commons Attribution Non-Commercial License (http://creativecommons.org/licenses/by-nc/4.0/). 
Kim WB, et al. • Burned-Out Testicular Choriocarcinoma with Retroperitoneal and Liver Metastases
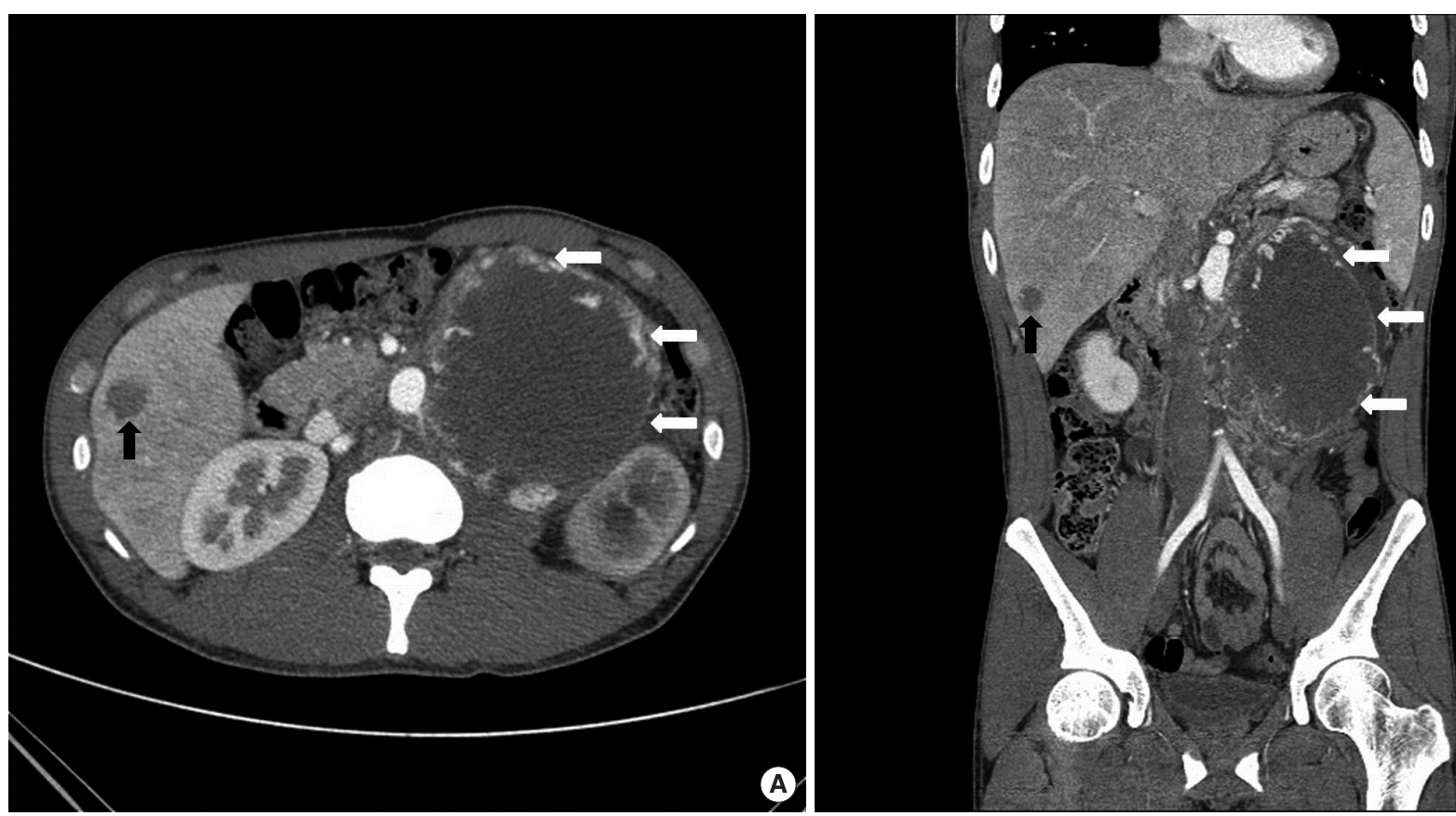

(B)

Fig. 1. (A, B) Computed tomography scan showing a huge retroperitoneal mass (white arrows) and a low-density liver lesion (black arrow). Left renal parenchymal enhancement was reduced by compressing the retroperitoneal mass.
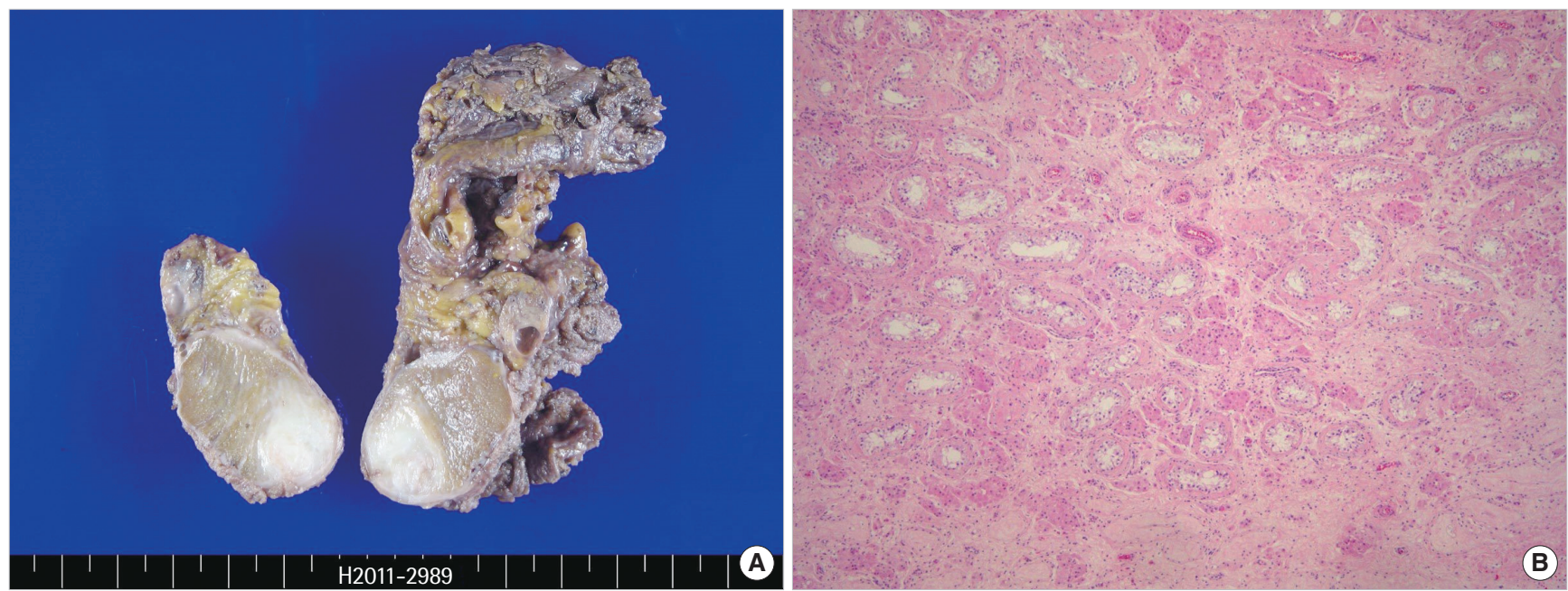

Fig. 2. (A) Gross findings of a white-to-tan colored and well-delineated nodular mass of the left testis measuring $1.5 \times 1.5 \mathrm{~cm}$. (B) Microscopic findings of the left testis showed testicular atrophy, as demonstrated by shrunken seminiferous tubules with decreased spermatogenesis and aggregation of Leydig cells (H\&E, $\times 40)$.

consistent with metastatic choriocarcinoma. Thus, a diagnosis of spontaneous regression of a germ cell tumor was made. Later, we performed two cycles of BEP (bleomycin+etoposide+cisplatin) chemotherapy, but improvement was not observed on follow-up $\mathrm{CT}$, so we changed the treatment to EMA-CO (etoposide+methotr exate+actinomycin+cyclophosphamide+oncovin). The sizes of the retroperitoneal and hepatic mass lesions decreased, and tumor markers normalized ( $\beta$-hCG, $0.3 \mathrm{mIU} / \mathrm{mL}$ ) after seven EMA-CO chemotherapy cycles. We then completely excised the retroperitoneal and hepatic mass lesions, and complete regression was found in both specimens. The patient has been followed for 9 months in the out-patient clinic under a diagnosis of complete remission.

\section{DISCUSSION}

A few reports from the early 20 th century suggested that testic- 


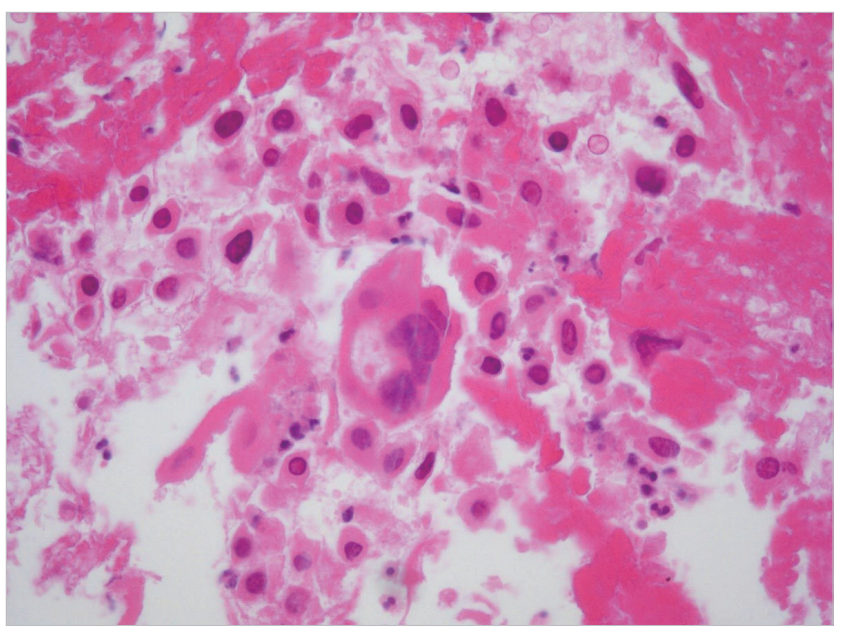

Fig. 3. Microscopic findings of the liver needle biopsy showed both neoplastic cytotrophoblasts and syncytiotrophoblasts. These findings were consistent with metastatic choriocarcinoma $(H \& E, \times 200)$.

ular germ cell tumors may undergo spontaneous regression. Friedman [2] reported that 23 of 29 patients with primary retroperitoneal germ cell tumors had either regressive changes or an overt tumor of the testis. Ha et al. [3] reported metastatic retroperitoneal seminoma with the 'burned-out' phenomenon in testis, but no case has been reported in Korea, with the exception of the present case.

Several immunological and ischemic mechanisms have been suggested to explain this spontaneous regression, but immunological mechanisms appear to be the most likely [4]. This immunological mechanism hypothesis for the regression of a primary testicular tumor suggests that common tumor antigens can be recognized after repeated exposure by cytotoxic T lymphocytes, which are subsequently replaced by fibrosis. However, no consensus exists.

Patients with extragonadal germ cell tumors and spontaneous regression in the testis usually show various clinical symptoms. In this case, our patient complained of left upper quadrant pain and dyspepsia. Although we suspected a testis-originating extragonadal germ cell tumor because of the elevated $\beta$-hCG, it was insufficient for the diagnosis. Scrotal ultrasonography may be helpful in such a situation. Comiter et al. [5] reported detection of intratesticular lesions by ultrasonographic examination in patients with retroperitoneal extragonadal germ cell tumors. In particular, microlithiasis is a recognized risk factor and reflects a background upon which testicular germ cell tumors commonly develop [6], but not in this case.
Radical orchiectomy with spontaneously regressing testis is controversial. Although current chemotherapy is largely effective against all type of metastatic testicular germ cell tumors, it is often not effective against primary testicular tumors because of the 'blood-testis barrier' [7]. Culine et al. [8] reported that identifying a primary testicular tumor in patients with an extragonadal germ cell tumor is important because it carries the danger of persistent testicular malignancy in up to $50 \%$ of patients, despite systemic chemotherapy. Therefore, a radical orchiectomy for an intratubular germ cell tumor in patients with the 'burned-out' phenomenon should be considered.

Cisplatinum-based chemotherapy after radical orchiectomy of a primary testicular tumor is the basis of the treatment regimen [9]. The prognosis of patients with an seminomatous extragonadal germ cell tumor in the retroperitoneum is better than that of patients with a nonseminomatous extragonadal germ cell tumor. Schmoll [10] reported that the majority of extragonadal germ cell tumors were of a nonseminomatous pathological type, which have a poor prognosis. If a residual retroperitoneal mass classified higher than stage IIc is present after chemotherapy in cases of nonseminomatous germ cell tumor, it is mandatory to excise the retroperitoneal mass and perform a tumorectomy. Although the patient in our case had a non-seminomatous extragonadal germ cell tumor, complete remission was achieved by completely excising the retroperitoneal and hepatic masses after chemotherapy.

The 'burned-out' phenomenon, known as spontaneous regression of a primary testicular tumor, is difficult to recognize and has been incompletely characterized by physicians. Extragonadal germ cell tumors in the retroperitoneum, liver, and other organs should be considered for the 'burned-out' phenomenon. Additional studies are needed to identify the exact mechanism and further delineate the nature of the 'burned-out' phenomenon.

\section{REFERENCES}

1. Azzopardi JG, Mostofi FK, Theiss EA. Lesions of testes observed in certain patients with widespread choriocarcinoma and related tumors: the significance and genesis of hematoxylin-staining bodies in the human testis. Am J Pathol 1961;38:207-25.

2. Friedman NB. The comparative morphogenesis of extragenital and gonadal teratoid tumors. Cancer 1951;4:265-76.

3. Ha HK, Jung SG, Park SW, Lee W, Lee SD, Chung MK. Retroperitoneal seminoma with the 'burned out' phenomenon in the testis. Korean J Urol 2009;50:516-9.

4. Tefany FJ, Barnetson RS, Halliday GM, McCarthy SW, McCarthy WH. Immunocytochemical analysis of the cellular infiltrate in primary re- 
gressing and non-regressing malignant melanoma. J Invest Dermatol 1991;97:197-202.

5. Comiter CV, Renshaw AA, Benson CB, Loughlin KR. Burned-out primary testicular cancer: sonographic and pathological characteristics. J Urol 1996;156:85-8.

6. Bach AM, Hann LE, Hadar O, Shi W, Yoo HH, Giess CS, et al. Testicular microlithiasis: what is its association with testicular cancer? Radiology 2001;220:70-5.

7. Greist A, Einhorn LH, Williams SD, Donohue JP, Rowland RG. Pathologic findings at orchiectomy following chemotherapy for disseminated testicular cancer. J Clin Oncol 1984;2:1025-7.

8. Culine S, Theodore C, Terrier-Lacombe MJ, Droz JP. Primary chemotherapy in patients with nonseminomatous germ cell tumors of the testis and biological disease only after orchiectomy. J Urol 1996;155:1296-8.

9. Geldart TR, Simmonds PD, Mead GM. Orchidectomy after chemotherapy for patients with metastatic testicular germ cell cancer. BJU Int 2002; 90:451-5.

10. Schmoll HJ. Extragonadal germ cell tumors. Ann Oncol 2002;13 Suppl 4:265-72. 